\title{
EDITORIAL
}

\section{Robotic prostatectomy: hit or myth?}

$\mathrm{R}$ adical prostatectomy $(\mathrm{RP})$ is arguably the most subtle and challenging operation that urologists perform. Any well-trained urologist can remove the prostate without life-threatening complications, but the ideal procedure would remove the prostate, seminal vesicles, and pelvic lymph nodes (when indicated) with negative surgical margins, no recurrence of cancer, and complete recovery of urinary and erectile function. Achieving this 'trifecta' is the ultimate challenge to modern urologic surgeons, and none are universally successful (Saranchuk, J. W. et al. J. Clin. Oncol. 23, 4146-4151; 2005).

For surgeons struggling to perform RP, the da Vinci ${ }^{\circledR}$ surgical system (Intuitive Surgical, Sunnyvale, CA, USA) promises an easier way to achieve the ideal result. The robot offers magnified three-dimensional visualization, remarkable precision and steadiness, and ease of use. The surgeon can sit at a console and use familiar hand motions to perform a complex laparoscopic resection and reconstruction as a 'minimally invasive' procedure. When this remarkable new tool was introduced, the manufacturer advertised, surgeons assumed, and patients were led to believe that their cancer would be cured with fewer complications and better return of sexual and urinary function (Schroek, F. R. et al. Eur. Urol. 54, 785-793; 2008). Robotic RP was perceived as the 'killer app' that mandated a robot in every major medical center and solidified the role of robotics in surgery. Today, $40-60 \%$ of all RPs in the US are performed robotically. So, has robotic prostatectomy met its expectations?

Before the robot became popular, laparoscopic RP had become an established alternative to open RP and seemed to offer distinct benefits: the incision was smaller, blood loss less and hospital stays shorter-all expected features of a minimally invasive operation. However, early claims of improved cancer control and better urinary and sexual function have never been substantiated. In fact, even with an experienced surgeon, incontinence is more likely after laparoscopic than open surgery. As for being 'minimally invasive', patients who undergo the laparoscopic procedure are actually more likely to return to the emergency room, hospital or operating room to correct a complication (Touijer, K. et al. J. Urol. 179, 1811-1817; 2008). Performed free hand, laparoscopic RP is difficult to master and requires many years of training. The learning curve is longer than for open RP (Vickers, A. J. et al. Lancet Oncol. 10, 475-480; 2009). Robot-assisted laparoscopic prostatectomy (RALP) seemed to have a much shorter learning curve. Single-surgeon case series touted the superior results of RALP, although these benefits were not seen in larger, single-institution comparative analyses.

No prospective randomized trials or multi-institutional series have examined whether the cost of robotic technology is worth the presumed benefits. The recent study by $\mathrm{Hu}$ et al. (Hu, J. C. et al. JAMA 302, 1557-1564; 2009) is the first nationwide, US population-based comparison of outcomes after open and laparoscopic (mainly robotic) RP. Chamie and Litwin provide their thoughts on the results in this issue of Nature Reviews Urology (page 121). The study confirmed shorter hospital stays and fewer blood transfusions and anastomotic strictures with RALP, but there was no difference in perioperative mortality. Although respiratory and surgical complication rates were lower, genitourinary complications, incontinence and erectile dysfunction were more common after RALP. In a similar, independent study of an earlier national US cohort, Lowrance and colleagues found no difference in perioperative morbidity or mortality and no difference in recovery of urinary function between open and laparoscopic RP (W. Lowrance, personal communication).

These results contradict the argument that laparoscopic RP, robotically assisted or not, is 'minimally invasive. The incision might be smaller but the risk of serious complications is just as great. Most disturbing of all is the lack of evidence to support the idea that using a surgical robot confers any advantage in the key outcomes that drive patients to seek robotic surgery in the first place; the chances of curing cancer or recovering urinary and sexual function are no better, and might be worse.

It is time for surgeons and the public to give up the notion that robotic surgery is automatically superior and to focus on factors that can actually improve surgical outcomes. The serious risks of RP, by any technique, should warn patients with favorable cancers away from surgery in favor of active surveillance, which is a safer alternative. When surgery is indicated, the results depend on the skill and experience of the surgeon, not the instrument s/he uses. Surgeons committed to achieving better results should pay less attention to technology and more to accurately measuring their outcomes to learn how best to improve their results. Better teaching tools, such as surgical simulators, could help shorten the learning curve for $\mathrm{RP}$ by any technique. It is time to abandon the hyperbole and aggressive marketing that have been an embarrassing distraction from our real goal: improving outcomes for patients with prostate cancer and working towards the day when all patients who require $\mathrm{RP}$ win the trifecta.

doi:10.1038/nrurol.2010.13

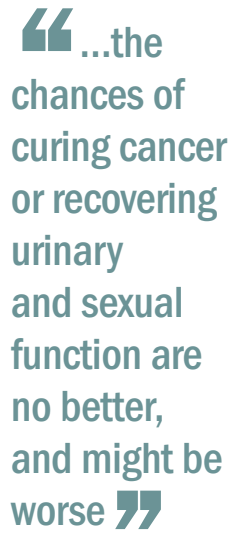

Peter T. Scardino is the Editor-in-Chief of Nature Reviews Urology.

Competing interests The author declares no competing interests. 